\title{
Extension of internal ratio of a line segment into complex values and its application to the generalization of Menelaus' theorem
}

\author{
Masayuki Ichikawa ${ }^{1}$ Naoyasu Kita ${ }^{2,{ }^{*}}$ Hiroto Matsumoto ${ }^{3}$ Yoshihisa Nakamura $^{4}$ \\ ${ }^{1}$ Oki Gakuen High School, Takeshita 2-1-33, Hakata-ku, Fukuoka, 812-0895 Japan \\ 2,3,4 Kumamoto University, Kurokami 2-39-1, Chuo-ku, Kumamoto, 860-8555 Japan \\ *Corresponding author. Email: nkita@kumamoto-u.ac.jp
}

\begin{abstract}
In mathematics and mathematics education, ranges of application are sometimes generalized. This article studies the generalization of the concept on internally dividing points. The ratios of internally dividing points are usually considered in real numbers since the real numbers are naturally corresponding to the length of line segments. The aim of this article is to extend the range of such ratios into the complex numbers. After defining the complex-valued ratio, we shall apply it to the generalization of Menelaus' theorem which is famous in elementary geometry.
\end{abstract}

Keywords: Line segment, Menelaus' theorem.

\section{INTRODUCTION}

When we were pupils of elementary schools, the concept of partition of a line segment was taught with the internal ratio - for example, divide a line segment at ratio of $1: 2$ or $3: 4$. It sounds funny if one says "divide a line segment at ratio of $i: 1+i$ ", where $i$ denotes the wellknown imaginary unit. Which point should be pointed out? To be honest, does it make a sense when the concept of the ratio is extended into complex numbers? The aim of this article is to introduce a definition of complexvalued internal ratios in reasonable way.

We know that the extension of the number field was often conducted in the educational process. Looking back our educational career, we know that, in the lower course of elementary schools, multiplication of natural numbers is instructed. In the middle course, a number 0 is included in the multiplication, and, in the upper course, positive fractional numbers are considered. After the enrollment in junior high school, the computation treats not only negative quotient numbers but also whole real numbers, in the style of instructing to our intuition. In senior high school, it is extended further into the complex numbers. Such extension of number field is not conducted only in the case of four arithmetic operations. Recalling the exponential function learned in junior and senior high schools, we remember that its domain is considered firstly in natural numbers. After that, it is extended into integers, quotient numbers and real numbers, of course, without rigorous definition. Finally most students of science are impressed, in the under-graduate course of universities, to see that the domain of the exponential function can be extended into the complex numbers and to know that there is an attractive relation among $0,1, \pi$ and $e$, i.e., $e^{\pi i}+1=0$ found by Euler.

As we see above, the extension of number field is found to be performed in the categories of algebra and calculus. Then it is natural to think about such extension in geometry. Following this motivation, we focus on the inertial ratio of a line segment. When one says "find a point internally dividing a line segment $\mathrm{AB}$ at ratio of $2: 1$ ", we will easily answer to this question by saying "it is a point $\mathrm{C}$ on the line segment $\mathrm{AB}$, which possesses the ratio of line segments $\mathrm{AC}$ and $\mathrm{CB}$ as $2: 1$ ". Why is it possible to find a correct answer? That is because "the length" of the line segments has so much to do with the ratio when it consists of a pair of real numbers.

Then, what if one says "find a point internally dividing $\mathrm{AB}$ at ratio of $i: 1+i$ "? If we were conservative and fixed our mindset such as the ratio must be related with the length of segments, this question would sound so meaningless. Of course, it is difficult to find out any line segments of $i[\mathrm{~m}]$ and $1+i[\mathrm{~m}]$ long on $\mathrm{AB}$. There is no line segment of imaginary-numbervalued length. Nevertheless, in this article, we will 
somehow try to define an internally dividing point at ratio of complex numbers. Our extension of the concept on the ratio is based on the career we have obtained in mathematics education. - Mathematics sometimes breaks our fixed concept by applying "the properties" of numbers in surprising way.

Taking the exponential function as an example, we shall explain what the final sentence in the former paragraph means. We have the typical properties called the exponential law such that

$$
e^{m} e^{n}=e^{m+n},\left(e^{m}\right)^{n}=e^{m n}
$$

The proof of the above properties is easy if $m$ and $n$ are natural numbers. In high school mathematics, we assume that the property (1.1) still holds for integers $m$ and $n$, and we find that the definitions such as $e^{0}=1$, $e^{-m}=1 / e^{m}$ are reasonable. Besides, by assuming that (1.1) holds for quotient numbers $m$ and $n$, we see that the definition such as $e^{m / n}=\sqrt[n]{e^{m}}$ is reasonable. Applying the completeness of real numbers, we can define the exponential function for all real numbers. Remark that, for real number $x$, the function $e^{x}$ is represented by Taylor series, i.e.,

$$
e^{x}=1+\frac{1}{1 !} x+\frac{1}{2 !} x^{2}+\frac{1}{3 !} x^{3}+\cdots
$$

The property (1.2) allows us to substitute a complex number in to $x$. - The convergence of the series in (1.2) follows from the completeness of complex numbers. In such a way, the exponential function is defined for all complex numbers, despite it is meaningless if one tries to count $e$ 's complex-valued times. We want the readers to see that the properties like (1.1) and (1.2) contribute to extending the domain of the exponential function.

Turning back to our interest, we notice, from the above survey on the exponential function, that certain property on internally dividing points is expected to provide the way of defining the complex-valued ratio. In fact, we will apply a formula on the representation of internally dividing points. When two points $\mathrm{A}(a)$ and $\mathrm{B}(b)$ are given on the number line, and when they locate at the ends of a line segment, how is the internally dividing point of $\mathrm{AB}$ at ratio of $m: n$ described? This problem is easy to be solved. Everyone knows that

$$
c=\frac{n a+m b}{m+n}(1.3)
$$

provides the desired point. We will apply the property (1.3) to the extension of the ratio into the complex-valued one. See Definition 2.1. Other details are described in section 2 .

As a mathematical application of this extended inertial ratio, a variant of famous Menelaus' theorem in the elementary geometry will be considered. Set a triangle $\mathrm{ABC}$ on a plane. There are three points $\mathrm{D}, \mathrm{E}$ and $\mathrm{F}$ which internally divide $\mathrm{BC}, \mathrm{CA}$ and $\mathrm{AB}$ (resp.) at ratio of $s:(1-s), t:(1-t)$ and $u:(1-u)$ (resp.). - The description such as $s:(1-s)$ for the ratio is convenient since it stands for the externally dividing ratio if $s<0$ or $1<s$, as well as for the normal internally dividing ratio if $0<s<1$. Then Menelaus' theorem tells us that the points D, E and F lie on one straight line if and only if

$$
\frac{s}{1-s} \cdot \frac{t}{1-t} \cdot \frac{u}{1-u}=-1
$$

In section 3 , we shall study the extension of the real numbers $s, t$ and $u$ of (1.4) into some complex numbers $\lambda$, $\mu$ and $v$ (resp.). We proved that the necessary and sufficient condition of collinear D, E, F for any triangle $\mathrm{ABC}$ is

$$
\frac{\lambda}{1-\lambda} \cdot \frac{\mu}{1-\mu} \cdot \frac{v}{1-v}=-1
$$

and, in addition,

$$
\frac{\lambda}{1-\mu}, \frac{\mu}{1-v}, \frac{v}{1-\lambda} \in \mathbf{R} . \text { (1.6) }
$$

(See Theorem 3.1.) The presence of (1.6) is remarkable. It is meaningless if $\lambda, \mu$ and $v$ are real. However, once we use complex ratio, the additional condition (1.6) is inevitable. Under the absence of (1.6), Theorem 3.1 fails. (See Fig.3.2.)

The generalization of Menelaus' theorem has been conducted in several directions. In [3], a variant of Menelaus' theorem is considered for triangles on a sphere. If one does not want to stick to triangles, refer to [2]. - It generalizes Menelaus' theorem for $n$-gons. Furthermore, there is no need to restrict the number of dividing points into 3. In [1], generalized Menelaus' theorem is considered for 6 dividing points. As far as the authors are concerned, the complex-valued ratios have not been treated for the generalization of Menelaus' theorem. Therefore Theorem 3.1 seems to be new.

\section{DEFINITION OF COMPLEX-VALUED RATIO}

We proceed in heuristic way. We have already learned how to compute the dividing point on a line segment. Let $\mathrm{A}$ and $\mathrm{B}$ be the end points of a line segment lying on the $x$-axis. The coordinates of $\mathrm{A}$ and $\mathrm{B}$ are $x=a$ and $x=b$ respectively. A point denoted by $\mathrm{C}$ ( $x=c$ ) divides the line segment $\mathrm{AB}$ at ratio of $m: n$, where $m$ and $n$ are positive real numbers at present. Then it is well-known that the coordinate $c$ is computed by

$$
c=\frac{n a+m b}{m+n}
$$


Our idea to extend the number field for $m$ and $n$ is just substituting complex numbers in the above expression. However, in that process, the value of $c$ becomes a complex number in general, and it goes away from the $x$-axis. Thus we need putting the line segment in the complex plane, and also regarding the end points $\mathrm{A}$ and $\mathrm{B}$ as points in that complex plane : treat the coordinates $a$ and $b$ as complex numbers. Now we are ready for defining the complex-valued inertial ratio of a line segment.

\section{Definition 2.1 (Complex-valued inertial ratio of a line segment)}

Let $\mathrm{A}(\alpha)$ and $\mathrm{B}(\beta)$ be disjoint points in the complex plane. Then, for complex numbers $\mu$ and $v$ satisfying $\mu+v \neq 0$, we say that a point $\mathrm{C}(\gamma)$ is internally dividing the line segment $\mathrm{AB}$ at ratio of $\mu: v$ if and only if

$$
\gamma=\frac{v \alpha+\mu \beta}{\mu+v}
$$

For example, when we take A (0) and B (1) in the complex plane with $\mu=i$ and $v=1+i$, the internally dividing point $\mathrm{C}(\gamma)$ of the line segment $\mathrm{AB}$ at ratio of $i: 1+i$ is computed in such a way that $\gamma=$ $\frac{(1+i) \times 0+i \times 1}{i+(1+i)}=\frac{2}{5}+\frac{1}{5} i$, and it is sketched in Fig. 1 .

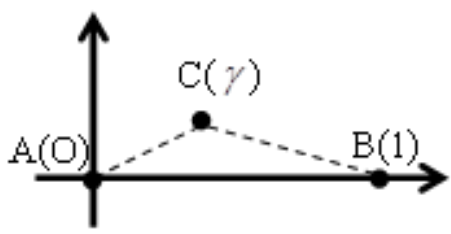

Figure 1 internally dividing point.

In Definition 2.1, one may be confused to look at a word "internally", since the point $\mathrm{C}(\gamma)$ sometimes steps away from the line segment AB. However, in this article, we keep using such a word in order to strongly leave an extended concept of the inertial point in our mind, and also in order to avoid the abuse of new words.

It is not so clear from Definition 2.1 how to find an internally dividing point of a line segment. In addition, it does not look so efficient to struggle in the computation every time a complex-valued ratio $\mu: v$ is given. To find the internally dividing point more easily, noticing the next fundamental properties makes our approach so simple.

\section{Proposition 2.2 (Properties of internally dividing points)}

Let $\mathrm{A}(\alpha)$ and $\mathrm{B}(\beta)$ be disjoint points in the complex plane, and let $\mu, v$ be complex numbers satisfying $\mu+v \neq 0$. Denote the internally dividing point of the line segment $\mathrm{AB}$ at ratio of $\mu: v$ by

$$
f_{\mu, v}(\alpha, \beta)=\frac{v \alpha+\mu \beta}{\mu+v}
$$

Then the following two statements holds.

(i) (Property on the translation) For any $\gamma \in \mathbf{C}$, we have

$$
f_{\mu, v}(\alpha+\gamma, \beta+\gamma)=f_{\mu, v}(\alpha, \beta)+\gamma
$$

(ii) (Property on the Rotation and Magnification) For any $\gamma \in \mathrm{C} \backslash\{0\}$, we have

$$
f_{\mu, v}(\gamma \alpha, \gamma \beta)=\gamma f_{\mu, v}(\alpha, \beta)
$$

Proof. Since the proof follows from a direct computation, we omit the detail.

Proposition 2.2 suggests that the location of the internally dividing point $f_{\mu, v}(\alpha, \beta)$ is deeply related with $f_{\mu, v}(0,1)$. In other words, noting that $\alpha=(\beta-$ $\alpha) \cdot 0+\alpha$ and $\beta=(\beta-\alpha) \cdot 1+\alpha$, we see, due to Proposition 2.2, that $f_{\mu, v}(\alpha, \beta)=(\beta-\alpha) f_{\mu, v}(0,1)+$ $\alpha$. The identity indicates that the internally dividing point of $\mathrm{AB}$ is accomplished by the combination of translation, rotation and magnification of $f_{\mu, v}(0,1)$.

Let us see the point denoted by $f_{\mu, v}(0,1)$. It is rewritten as

$$
f_{\mu, v}(0,1)=\frac{\mu}{\mu+v}:=\varsigma
$$

One may take it as $f_{\mu, v}(0,1)$ is an internally dividing point of the line segment connecting 0 and 1 at ratio of $\zeta: 1-\zeta$. Then we have the following procedure for detecting an internally dividing point of $\mathrm{AB}$.

\section{Procedure for detecting an internally dividing point}

(Step 1) Once complex numbers $\mu$ and $v$ (with $\mu+v \neq 0$ ) are given, compute $\mu /(\mu+v)$ and denote it by $\zeta$.

(Step 2) Place a virtual origin (denoted by $\langle\mathrm{O}\rangle$ ) at $\mathrm{A}(\alpha)$, and a virtual unit (denoted by $\langle 1>$ ) at $\mathrm{B}(\beta)$. Imagine a virtual complex plane with $\langle\mathrm{O}\rangle$ as its origin and with the line connecting $\langle\mathrm{O}\rangle$ and $\langle 1\rangle$ as a virtual real axis. - Note that the virtual imaginary axis is pointing in the direction 
anti-clockwise rotated by $\pi / 2$ [rad] from the virtual real axis. We denote this virtual complex plane by $\langle\mathbf{C}\rangle$. (See Fig.2.)

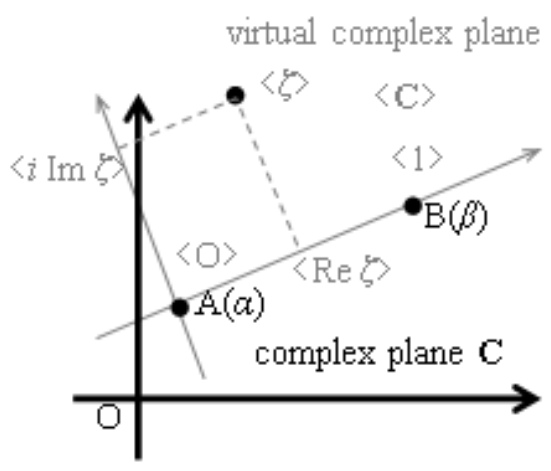

Figure 2 virtual complex plane

(Step 3) On the virtual complex plane $\langle\mathbf{C}\rangle$, dot a point denoted by $\zeta$ (or it must be denoted by $\langle\zeta\rangle$ ) obtained in (Step 1). Regard this point as a point of the original complex plane $\mathbf{C}$. Then this is the desired internally dividing point of $\mathrm{AB}$.

We must always take care of the direction of the virtual imaginary axis. It is pointing to our left when we are facing in the positive direction of the real axis.

\section{MENELAUS' THEOREM WITH COMPLEX-VALUED INTERNALLY DIVIDING RATIO}

Speaking of geometric theorems treating the internally dividing ratios, one may always take up Menelaus' theorem as an example. In this section, we shall prove a variant of Menelaus' theorem with the complex-valued ratio as in Definition 2.1. Unlike the real-valued case, additional relation of ratios appears in the collinear condition.

\section{Theorem 3.1 (Menelaus' theorem with complex- valued ratio)}

Let $\lambda, \mu$ and $v$ be complex numbers different from 0 and 1. Assume that there are three distinct points $\mathrm{A}\left(z_{A}\right)$, $\mathrm{B}\left(z_{B}\right)$ and $\mathrm{C}\left(z_{C}\right)$ on the complex plane. Let $\mathrm{D}, \mathrm{E}$ and $\mathrm{F}$ be the internally dividing points of the line segment $\mathrm{BC}, \mathrm{CA}$ and $\mathrm{AB}$ (resp.) at complex-valued ratio of $\lambda:(1-\lambda)$, $\mu:(1-\mu)$ and $v:(1-v)$ (resp.). Then $\mathrm{D}, \mathrm{E}$ and $\mathrm{F}$ are collinear for any distinct complex numbers $z_{A}, z_{B}$ and $z_{C}$ if and only if both

$$
\frac{\lambda}{1-\lambda} \cdot \frac{\mu}{1-\mu} \cdot \frac{v}{1-v}=-1
$$

and $\frac{\lambda}{1-\mu}, \frac{\mu}{1-\gamma}, \frac{v}{1-\lambda} \in \mathbf{R}$

hold.

Remark. For example, the complex values $\lambda=-1+i$, $\mu=i$ and $v=1-(1 / 2) i$ satisfy both (3.1) and (3.2). Take $\mathrm{A}(2+4 i), \mathrm{B}(0)$ and $\mathrm{C}(4+i)$. Then, following Definition 2.1, we have $\mathrm{D}(-5+3 i), \mathrm{E}(1-i)$ and $\mathrm{F}(-2+i)$ as complex-valued internally dividing points, which are surely collinear. (See Fig. 3)

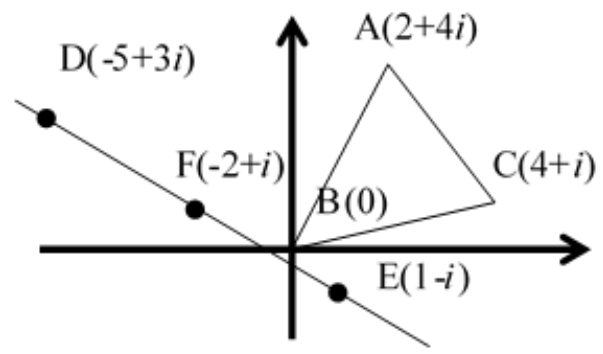

Figure 3 D, E and F are collinear.

Remark. When we consider real-valued ratios, (3.2) is automatically satisfied and so it does not look crucial. Nevertheless, the additional condition (3.2) becomes important when we consider complex-valued ratios. For example, $\lambda=1+i, \mu=1+i$ and $v=(1-2 i) / 5$ satisfy only (3.1). In this case, the internally dividing points D, E and F are not always collinear. In Fig. 3.2, the situation is indicated for $\mathrm{A}(5+25 i), \mathrm{B}(5+5 i)$ and $\mathrm{C}(25+15 i)$. Remark here that a quite special triangle possibly shows collinear result on its three internal dividing points even though (3.2) fails. We want to say that (3.2) ensures collinear results universally for all triangles.

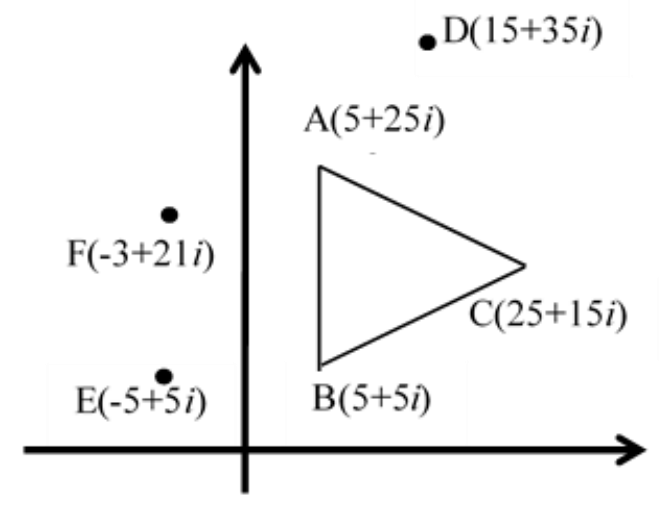

Figure 4 D, E and F are not collinear. 
Now let us prove Theorem 3.1.

Proof of Theorem 3.1. Denote the points D, E and F in the complex plane by complex numbers $z_{D}, z_{E}$ and $z_{F}$. Then, following Definition 2.1, we have

$$
\begin{aligned}
& z_{D}=(1-\lambda) z_{B}+\lambda z_{C}, \quad z_{E}=(1-\mu) z_{C}+\mu z_{A}, \\
& z_{F}=(1-v) z_{A}+v z_{B}
\end{aligned}
$$

Since $\mathrm{D}, \mathrm{E}$ and $\mathrm{F}$ are assumed to be collinear for any distinct $z_{A}, z_{B}$ and $z_{C}$, we see that there exists some real number $r$ such that

$$
z_{F}-z_{D}=r\left(z_{E}-z_{D}\right)
$$

It is easy to see that this is equivalent to

$$
\begin{aligned}
& \lambda\left(z_{B}-z_{C}\right)+(v-1)\left(z_{B}-z_{A}\right) \\
& =r\left((\lambda-1)\left(z_{B}-z_{C}\right)+\mu\left(z_{A}-z_{C}\right)\right) .
\end{aligned}
$$

Let $w=\left(z_{B}-z_{C}\right) /\left(z_{A}-z_{C}\right)-$ Since the points A, B and $\mathrm{C}$ form a triangle, the $w$ varies in the range of $w \notin \mathbf{R}$. Then $w$ is allowed to take all complex values except for 0 . From the identity (3.3), it follows that

$$
\frac{(\lambda+v-1) w-(v-1)}{(\lambda-1) w+\mu}=r .
$$

Note that, at present, the $r$ on the right hand side of (3.4) is possibly dependent on $w$. However, we can deny such dependence due to the theory of complex analysis. Namely, remark that the left hand side of (3.4) is a holomorphic function on $\mathbf{C} \backslash(\mathbf{R} \cup\{\mu /(1-\lambda)\})$. Following Cauchy-Riemann's equation, we can say that the $r$ must be a real constant independent of $w$. Now, multiplying $(\lambda-1) w+\mu$ on both hand side of (3.4), and matching the coefficients of $w$, we have

$$
\frac{\lambda+v-1}{\lambda-1}=r, \frac{1-v}{\mu}=r
$$

By (3.5), we have $(\lambda+v-1) \mu=(\lambda-1)(1-v)$. We write this identity in such a way that

$$
(\lambda+v-1-\lambda v+\lambda v) \mu=-(1-\lambda)(1-v),
$$

which is equivalent to

$$
-(1-\lambda)(1-v) \mu+\lambda \mu v=-(1-\lambda)(1-v)
$$

Then (3.1) follows. Looking at (3.5) again, we find that $v /(1-\lambda)$ and $\mu /(1-v)$ are real. By making use of (3.1), $\lambda /(1-\mu)$ is also real and hence (3.2) follows.

On the contrary, when (3.1) and (3.2) are assumed, it suffices to show that the left hand side of (3.4) takes real value for any complex $w$. By (3.2), we let

$$
\frac{\lambda}{1-\mu}=s \in \mathbf{R}, \frac{\mu}{1-v}=t \in \mathbf{R} .
$$

From (3.1), it follows that

$$
\text { st } v=-(1-\lambda)
$$

Applying (3.6) to the above identity, we see that

$$
\begin{aligned}
s t v & =-(1-s(1-\mu)) \\
& =s-1-s \mu \\
& =s-1-s t(1-v) .
\end{aligned}
$$

Since stv's on both hand sides are canceled, we have a relation of $s$ and $t$, i.e.,

$$
t=\frac{s-1}{s}
$$

We next deform $\lambda+v-1$ which appears in (3.4). By (3.7), we see that

$$
\lambda+v-1=(\lambda-1)\left(1+\frac{1}{s t}\right)
$$

By (3.8), we know that $s t=s-1$, and so we have

$$
\lambda+v-1=(\lambda-1) \cdot \frac{s}{s-1}=\frac{\lambda-1}{t} .
$$

By (3.9) and the second identity of (3.6), the left hand side of (3.4) is rewritten as

$$
\frac{(\lambda+v-1) w-(v-1)}{(\lambda-1) w+\mu}=\frac{(\lambda-1) w+\mu}{(\lambda-1) w+\mu} \cdot \frac{1}{t}=\frac{1}{t} \in \mathrm{R}
$$

As a result, the three points D, E and F are collinear. The proof is complete.

\section{CONCLUSION}

We can apply the property of the internally dividing point of $A B$ at ratio of $m: n$ to the extension of the ratio into the complex-valued one. To expand internally dividing points we must put the line segment in the complex plane, and also regarding the end points $\mathrm{A}$ and $\mathrm{B}$ as points in that complex plane: treat the coordinates and as complex numbers. Properties of internally dividing points for complex values are: translation, Rotation and Magnification. The identity indicates that the internally dividing point of $\mathrm{AB}$ is accomplished by the combination of translation, rotation and magnification. The Procedure for detecting an internally 
dividing point are: Once complex numbers $\mu$ and $v$ ) are given, compute $\mu /(\mu+v)$ and denote it by $\zeta$, second place a virtual origin at $\mathrm{A}()$, and a virtual unit at $\mathrm{B}(\mathrm{)}$.Third, On the virtual complex plane $\langle\mathrm{C}\rangle$, dot a point denoted by $\zeta$ (or it must be denoted by $<\zeta>$ ) obtained. Regard this point as a point of the original complex plane $C$. Then this is the desired internally dividing point of $\mathrm{AB}$.

As a mathematical application of this extended inertial ratio, a variant of famous Menelaus' theorem in the elementary geometry will be considered. Menelaus' theorem with complex-valued ratio explain that a quite special triangle possibly shows collinear result on its three internal dividing points. We want to say that (3.2) ensures collinear results universally for all triangles. We can deny such dependence due to the theory of complex analysis. Namely, remark that the left hand side of (3.4) is a holomorphic function on $\mathrm{C} \backslash(\mathrm{R} \cup\{\mu /(1-\lambda)\})$. F Cauchy-Riemann's equation, we can say that the $r$ must be a real constant independent of $w$. Now, multiplying ( $\lambda$ 1) $w+\mu$ on both hand side of (3.4), and matching the coefficients of $\mathrm{w}$

\section{ACKNOWLEDGMENTS}

The second author appreciate ICMMED organized in Pattimura University, Indonesia for giving him an opportunity to have a talk on the topic included in this article. The conference encouraged him to establish the theory of complex-valued ratio as an scientific article.

\section{REFERENCES}

[1] B.D.S. Blue McConnell, "A Six Point CevaMenelaus Theorem",arXiv:1403.0478 [math.MG].

[2] F. Smarandache, "Generalization of the Theorem of Menelaus Using a Self-Recurrent Method." Preparation des élèves marocains pour l'olympiade internationale des mathématiques de Paris - 1983, May 1983, Rabat, Morocco. ffhal-00495752f.

[3] E. W. Weisstein, "Menelaus' Theorem." From MathWorld - A Wolfram Web Resource 